\title{
Corrigendum: Tonic inhibition in dentate gyrus impairs long-term potentiation and memory in an Alzheimer's disease model
}

Zheng Wu, Ziyuan Guo, Marla Gearing \& Gong Chen

Nature Communications 5:4159 doi: 10.1038/ncomms5159 (2014); Published 13 Jun 2014; Updated 12 Sep 2014

The original version of this Article contained a typographical error in the spelling of 'Alzheimer's' in the title of the paper. This has now been corrected in both the PDF and HTML version of the Article. 